\title{
Detection of illicit online sales of fentanyls via Twitter
}

\section{[version 1; peer review: 3 approved]}

\author{
Tim K. Mackey (D)1-3, Janani Kalyanam³,4 \\ ${ }^{1}$ Department of Anesthesiology, University of California San Diego School of Medicine, San Diego, CA, 92093, USA \\ ${ }^{2}$ Division of Global Public Health, University of California, San Diego School of Medicine, San Diego, CA, 92093, USA \\ ${ }^{3}$ Global Health Policy Institute, San Diego, CA, USA \\ ${ }^{4}$ Department of Electrical and Computer Engineering, University of California, San Diego, San Diego, CA, 92093, USA
}

V1 First published: 02 Nov 2017, 6:1937

https://doi.org/10.12688/f1000research.12914.1

Latest published: 02 Nov 2017, 6:1937

https://doi.org/10.12688/f1000research.12914.1

\begin{abstract}
A counterfeit fentanyl crisis is currently underway in the United States. Counterfeit versions of commonly abused prescription drugs laced with fentanyl are being manufactured, distributed, and sold globally, leading to an increase in overdose and death in countries like the United States and Canada. Despite concerns from the U.S. Drug Enforcement Agency regarding covert and overt sale of fentanyls online, no study has examined the role of the Internet and social media on fentanyl illegal marketing and direct-to-consumer access. In response, this study collected and analyzed five months of Twitter data (from June-November 2015) filtered for the keyword "fentanyl" using Amazon Web Services. We then analyzed 28,711 fentanylrelated tweets using text filtering and a machine learning approach called a Biterm Topic Model (BTM) to detect underlying latent patterns or "topics" present in the corpus of tweets. Using this approach we detected a subset of 771 tweets marketing the sale of fentanyls online and then filtered this down to nine unique tweets containing hyperlinks to external websites. Six hyperlinks were associated with online fentanyl classified ads, 2 with illicit online pharmacies, and 1 could not be classified due to traffic redirection. Importantly, the one illicit online pharmacy detected was still accessible and offered the sale of fentanyls and other controlled substances direct-to-consumers with no prescription required at the time of publication of this study. Overall, we detected a relatively small sample of Tweets promoting illegal online sale of fentanyls. However, the detection of even a few online sellers represents a public health danger and a direct violation of law that demands further study.
\end{abstract}

\section{Keywords}

fentanyl, prescription drug abuse, digital surveillance, fentanyl, counterfeit, Twitter, social media, internet pharmacies

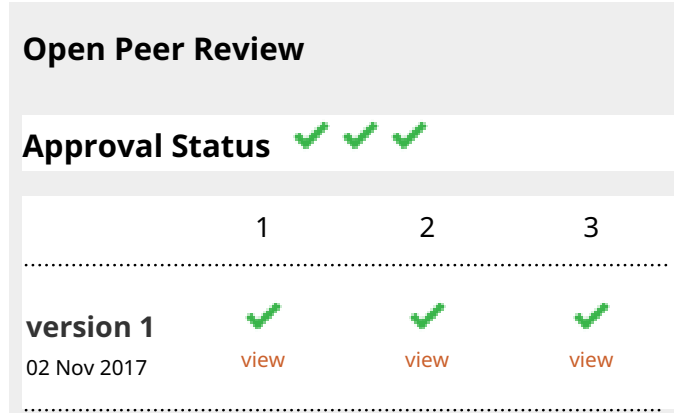

1. Marvin D. Shepherd, The University of Texas at Austin, Austin, USA

2. Róbert G. Vida, University of Pécs, Pécs, Hungary

3. Emilio Ferrara, University of Southern

California, Marina del Rey, USA

Any reports and responses or comments on the article can be found at the end of the article. 
This article is included in the Machine learning:

life sciences collection.

Corresponding author: Tim K. Mackey (tmackey@ucsd.edu)

Author roles: Mackey TK: Conceptualization, Data Curation, Formal Analysis, Funding Acquisition, Investigation, Methodology, Project Administration, Resources, Software, Supervision, Validation, Visualization, Writing - Original Draft Preparation, Writing - Review \& Editing; Kalyanam J: Conceptualization, Data Curation, Formal Analysis, Funding Acquisition, Investigation, Methodology, Project Administration, Resources, Software, Supervision, Validation, Visualization, Writing - Original Draft Preparation, Writing - Review \& Editing

Competing interests: TM is a non-compensated member of the academic advisory panel of the Alliance for Safe Online Pharmacies (ASOP), a 501(c)(4) social welfare organization engaged on the issue of illicit online pharmacies. There was no involvement of anyone other than the authors in the conception, design, collection, planning, conduct, analysis, interpretation, writing, and discussion to submit this work. Authors report no other financial relationships with any organizations that might have an interest in the submitted work.

Grant information: TM received funding for the data collection phase of this study from an ASOP pilot research grant exploring prescription drug abuse risks online. Though this pilot research grant supported the overall process of capturing data, the aims of the grant were not related to this study.

Copyright: $\odot 2017$ Mackey TK and Kalyanam J. This is an open access article distributed under the terms of the Creative Commons Attribution License, which permits unrestricted use, distribution, and reproduction in any medium, provided the original work is properly cited.

How to cite this article: Mackey TK and Kalyanam J. Detection of illicit online sales of fentanyls via Twitter [version 1; peer review: 3 approved] F1000Research 2017, 6:1937 https://doi.org/10.12688/f1000research.12914.1

First published: 02 Nov 2017, 6:1937 https://doi.org/10.12688/f1000research.12914.1 


\section{Introduction}

A fentanyl crisis is currently underway in the United States, characterized by an influx of counterfeit fentanyl-laced prescription drugs (e.g. Xanax ${ }^{\circledR}$, Norco ${ }^{\circledR}$, OxyContin $^{\circledR}$, and Oxycodone) now being advertised, sold and consumed by the public ${ }^{1}$. The result of this counterfeit infiltration into the U.S. drug supply chain has been an alarming increase in fentanyl-related overdose, deaths and seizures, due to illicitly produced products containing undeclared fentanyls and related fentanyl analogues/variants (e.g. acetyl fentanyl, butyrfentanyl, and furanylfentanyl.) ${ }^{1-3}$

Fueling this public health emergency is a global network of fentanyl producers and traffickers including countries such as China (where powdered fentanyls and other synthetic opiates are sold with pill presses or sold as precursors used to manufacture fentanyl), Mexico (where fentanyls are manufactured in clandestine laboratories and smuggled into the USA) and Canada (where fentanyls are also manufactured and sold locally). Unfortunately, criminals see an opportunity to sell cheap and deadly versions of fentanyl-laced pills to satiate demand driven by a national prescription opioid and heroin epidemic, with the U.S. Drug Enforcement Agency (DEA) estimating that a kilogram of fentanyl could generate $\$ 5-20$ million in retail counterfeit sales ${ }^{1}$.

Other studies have explored the public health consequences of counterfeit fentanyl and have argued for better surveillance and harm reduction approaches to curb consumption and demand $^{2,3}$. However, the potential impact and easy accessibility of fentanyls sold direct-to-consumer via the Internet and social media has not been examined. Other studies have identified illegal marketing and direct sales of other prescription controlled substances by illicit online pharmacies and the use of popular social media sites to promote these services ${ }^{4-10}$.

No study has assessed the role of Internet in fentanyl product promotion, despite concerns from the DEA about documented covert and overt sale of fentanyls online ${ }^{1}$. To better characterize these risks, we conducted a social media surveillance study using big data approaches to identify fentanyl promotion and sale via the popular microblogging platform Twitter.

\section{Methods}

Our data collection was part of a larger study exploring user behavior characteristics of illicit prescription drug abuse mediated by Twitter, with tweets collected over a five-month period (June-November 2015) filtered for the prescription drug abuserelated keywords, including the term "fentanyl." ${ }^{, 6,10}$ Data collection and analysis was carried out using a methodology combining Amazon Web Services (AWS) cloud computing virtual instances for data collection and machine learning algorithms to analyze tweets using both assisted and unassisted protocols used in previously published studies ${ }^{5,6,10}$.

Specifically, we used a Biterm Topic Model (BTM) that can identify underlying latent patterns or "topics" present in a large corpus of tweets ${ }^{6,11}$. Given the input corpus of text, and a predefined number $\mathrm{k}$, the model outputs a set of $\mathrm{k}$ latent topics present in the corpus. Each topic represents an underlying pattern associated with fentanyl twitter conversations that were then isolated and coded using human annotation.

The number of topics to be detected was set to 40 and the alpha and beta parameters of BTM were set to 1 and 0.01 respectively. The output of the BTM was set to display the top 10 words with the highest weight for each topic. Each topic was manually reviewed based on these words and classified into "news" and "online pharmacy" themes (results discussed below). We also used a keyword filtering process using common terms included in the text of tweets associated with illegal online drug promotion, also known as "selling arguments," (e.g. "buy", "discount", "price") in conjunction with the BTM outputs. Code used in this study is available from https://github.com/kjanani/ health_topicmodeling.

\section{Results}

Our study collected 28,711 fentanyl-related tweets during 2015, a period when the fentanyl crisis was escalating ${ }^{6,11}$. The majority of topics detected in the whole corpus of fentanyl-related tweets were news-related $(97.3 \%, n=27,940)$, detailing counterfeit fentanyl dangers reported in national and local media outlets.

After isolating Tweets related to news reports, we used a keyword filtering process in conjunction with machine learning to detect the subset of tweets associated with illegal online drug promotion. Using this approach, we detected $771(<1 \%$ of total $)$ tweets promoting the marketing and sale of fentanyls and other controlled substances online. These tweets were then manually annotated by the authors (inter-coder reliability kappa $=0.98$ ) to assess if they included a hyperlink enabling direct-to-consumer sale and purchase of illegal fentanyls. Nine unique tweets (not duplicates or retweets) and their associated hyperlinks were then identified for further analysis.

When examining website content of hyperlinks, 6 were associated with online classified ads, 2 with illicit online pharmacies, and 1 could not be classified due to traffic redirection. These results indicate some interesting trends. First, it appears that individual drug dealers use online classifieds ads to digitally advertise "street buys" of controlled substances (Example A, Figure 1). Additionally, one illicit online pharmacy that we detected and which is currently was still accessible at the time of initial publication of this study. offers the sale of fentanyls and other controlled substances direct-to-consumers with no prescription required (Example B). Its website purportedly offers Abstral ${ }^{\circledR} 800 \mathrm{mg}$ (fentanyl brand name) for $\$ 3.00$ per tablet, and based on further inspection of WHOIS data, has its internet domain registration identity and location masked by an Internet privacy service company.

We note that there are certain limitations to this study. First the study only examined online promotion and availability at a single point-of-time, as websites of this nature are often removed or become inactive. Additionally, because of the illegal nature of the websites identified, we did not purchase fentanyls and test them for authenticity and potency. Buying controlled substances and making payments to an illicit online pharmacy raises serious legal concerns and is generally illegal. 

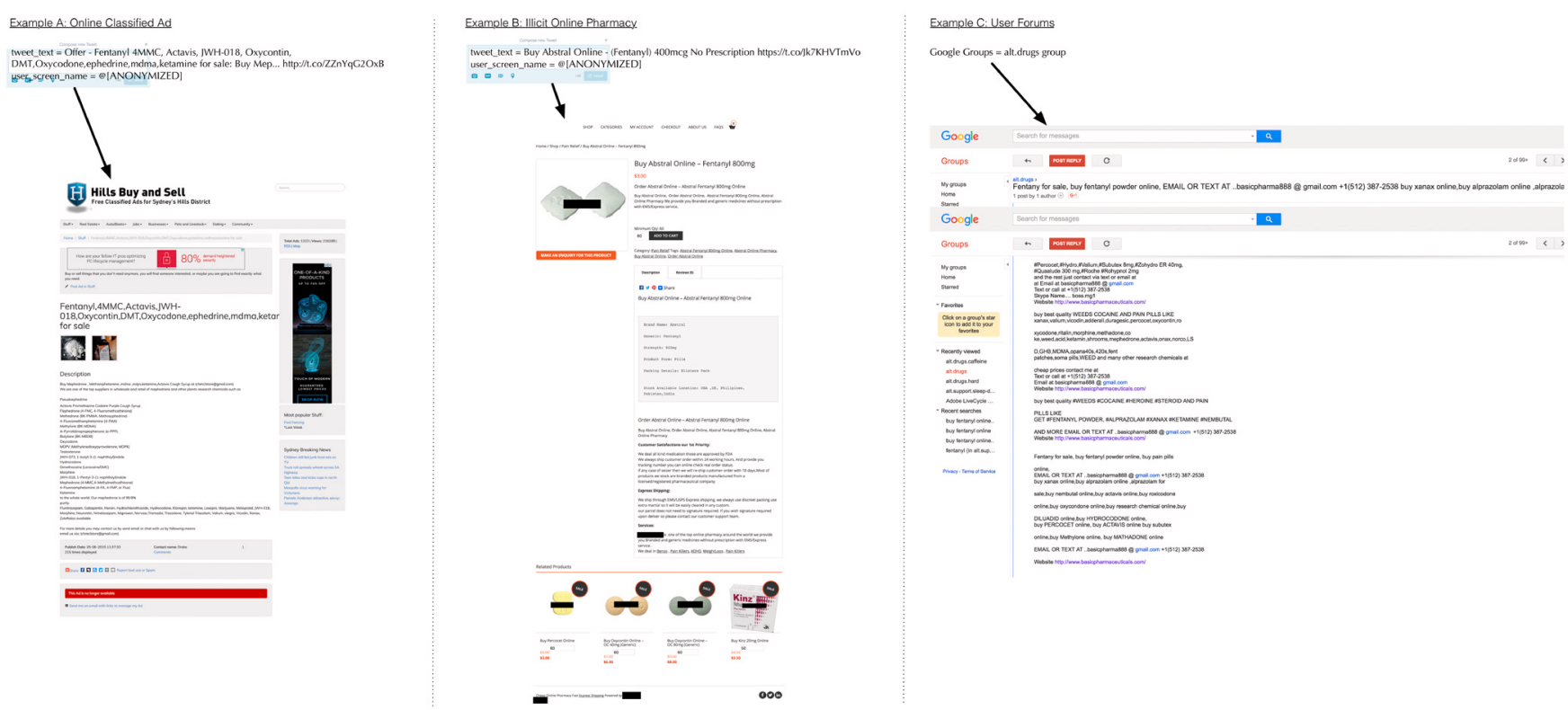

Figure 1. Screenshots of fentanyl promotion and sale via Twitter.

\section{Discussion}

Our results indicate that in our entire corpus of fentanyl-related tweets, direct-to-consumer sale of fentanyls occurred infrequently. This is not surprising, given the deadly nature and high potency of fentanyl, and that many victims may not actively seek its purchase $^{1,2}$. However, the presence of even a few online sellers is concerning, as these actions represent a clear violation of Federal law (e.g. Controlled Substances Act and the Ryan Haight Online Pharmacy Consumer Protection Act) and directly endanger the public. Importantly, these sites may only be the tip of the iceberg, as hyperlinks in one result directed us to online user forums (Google Groups) actively selling fentanyl online (Example C).

Our data collection occurred in late 2015, a period arguably at an early stage of the fentanyl crisis epidemiological curve, which may limit the generalizability of the results. Hence, this indicates that more research is needed to accurately characterize the dangers of the online environment in relation to counterfeit fentanyls. This includes longer term follow-up studies examining online promotion and access to fentanyl and interrogating other online platforms (including other social media sites, chat rooms/user forums, the deep web, and private sales facilitated by online and mobile technology).

\section{Data availability}

Raw datasets have not been made available per concerns regarding user information and confidentiality of publicly available processed data. This data is stored at the Global Health Policy Institute and is available upon request in a de-identified and aggregated dataset. Please contact the institute's general contact email (ghpolicyinstitute@gmail.com) for further information.

The code for BTM can be found at https://github.com/kjanani/ health_topicmodeling.

Archived code: https://doi.org/10.5281/zenodo.1038177'12

\section{Competing interests}

$\mathrm{TM}$ is a non-compensated member of the academic advisory panel of the Alliance for Safe Online Pharmacies (ASOP), a 501(c)(4) social welfare organization engaged on the issue of illicit online pharmacies. There was no involvement of anyone other than the authors in the conception, design, collection, planning, conduct, analysis, interpretation, writing, and discussion to submit this work. Authors report no other financial relationships with any organizations that might have an interest in the submitted work.

\section{Grant information}

TM received funding for the data collection phase of this study from an ASOP pilot research grant exploring prescription drug abuse risks online. Though this pilot research grant supported the overall process of capturing data, the aims of the grant were not related to this study. 
1. DEA: Counterfeit Prescription Pills Containing Fentanyls: A Global Threat [Internet]. dea.gov. 2016; [cited 2017 Feb 17].

Reference Source

2. Green TC, Gilbert M: Counterfeit Medications and Fentanyl. JAMA Intern Med. American Medical Association, 2016; 176(10): 1555-7.

PubMed Abstract | Publisher Full Text

3. Frank RG, Pollack HA: Addressing the Fentanyl Threat to Public Health. N Engl J Med. 2017; 376(7): 605-7.

PubMed Abstract | Publisher Full Text

4. Mackey TK, Liang BA, Strathdee SA: Digital social media, youth, and nonmedical use of prescription drugs: the need for reform. J Med Internet Res. 2013; 15(7): e143.

PubMed Abstract | Publisher Full Text | Free Full Text

5. Katsuki T, Mackey TK, Cuomo R: Establishing a Link Between Prescription Drug Abuse and Illicit Online Pharmacies: Analysis of Twitter Data. J Med Internet Res. 2015; 17(12): e280.

PubMed Abstract | Publisher Full Text | Free Full Text

6. Kalyanam J, Katsuki T, Lanckriet G, et al.: Exploring trends of nonmedical use of prescription drugs and polydrug abuse in the Twittersphere using unsupervised machine learning. Addict Behav. 2017; 65: 289-95. PubMed Abstract | Publisher Full Text
7. Raine C, Webb DJ, Maxwell SR: The availability of prescription-only analgesics purchased from the internet in the UK. Br J Clin Pharmacol. Blackwell Publishing Ltd, 2009; 67(2): 250-4

PubMed Abstract | Publisher Full Text | Free Full Text

8. Forman RF, Block LG: The Marketing of Opioid Medications without Prescription over the Internet. J Public Policy Mark. 2006; 25: 133-46. Publisher Full Text

9. U S Government Accountability Office HGG: INTERNET PHARMACIES: Federal Agencies and States Face Challenges Combating Rogue Sites, Particularly Those Abroad [Internet]. gao.gov. 2013, [cited 2016 Jan 11].

Those Abroad [Intern

10. Mackey TK, Kalyanam J, Katsuki T, et al.: Machine Learning to Detect Prescription Opioid Abuse Promotion and Access via Twitter. Am J Public Health: published online before print October 19, 2017. e1-e6. Health: published online before print Octo
PubMed Abstract | Publisher Full Text

11. Yan X, Guo J, Lan Y, et al.: A biterm topic model for short texts. The 22nd international conference. New York, New York, USA: ACM, 2013; 12. Publisher Full Text

12. Mackey T, Kalyanam J: Detection of Illicit Online Sales of Fentanyls via Twitter. Zenodo. 2017. Data Source 


\section{Open Peer Review}

\section{Current Peer Review Status:}

\section{Version 1}

Reviewer Report 18 December 2017

https://doi.org/10.5256/f1000research.14002.r28804

(C) 2017 Ferrara E. This is an open access peer review report distributed under the terms of the Creative Commons Attribution License, which permits unrestricted use, distribution, and reproduction in any medium, provided the original work is properly cited.

\section{Emilio Ferrara}

Information Sciences Institute, University of Southern California, Marina del Rey, CA, USA

This is a well written report on a timely and important research topic, directly connected to the current US opioid epidemic. Overall, the methodology is sound and the findings are interesting. One limitation of the data collection that could be addressed in future studies is the focus on one single keyword (fentanyl): a quick search using brand names (e.g., oxycodone, oxycontin) and slang terms / street names usually associated with this drug (e.g., ox, ocs) produce many additional hits, including at times associated with illicit person-to-person sales. The authors carried out a scrupulous investigation of the clearnet domains associated with the links discovered on Twitter - I wonder if a similar analysis could be carried out on the Dark Web (often associated with the illicit sale of drugs): this would require significant changes in the data collection infrastructure thus I can only expect it could be done in a follow up study.

Is the work clearly and accurately presented and does it cite the current literature? Yes

Is the study design appropriate and is the work technically sound? Yes

Are sufficient details of methods and analysis provided to allow replication by others? Yes

If applicable, is the statistical analysis and its interpretation appropriate? Yes

Are all the source data underlying the results available to ensure full reproducibility? Partly

Are the conclusions drawn adequately supported by the results? Yes 
Competing Interests: No competing interests were disclosed.

Reviewer Expertise: social networks, data science, machine learning, AI

I confirm that I have read this submission and believe that I have an appropriate level of expertise to confirm that it is of an acceptable scientific standard.

Reviewer Report 11 December 2017

https://doi.org/10.5256/f1000research.14002.r28806

(C) 2017 Vida R. This is an open access peer review report distributed under the terms of the Creative Commons Attribution License, which permits unrestricted use, distribution, and reproduction in any medium, provided the original work is properly cited.

\section{Róbert G. Vida}

Department of Pharmaceutics and Central Clinical Pharmacy, Faculty of Pharmacy, University of Pécs, Pécs, Hungary

An up-to-date and innovative methodological approach of a possible global problem of illegal trade of pharmaceuticals via the Internet is absolutely useful. The main messages are in accordance with the scientific literature.

The concern highlighted by the Dr. Marvin D. Shepherd regarding the dissonance between the introduction and the other parts of the publication is real and similarly I suggest the reconsideration and rewrite of that part. Also it would be advisable to include the comparison of the legal and illicit use and highlight the sources of fentanyl products or fentanyl containing products (outpatient and inpatient settings). Beside the counterfeit fentanyl crisis another possible public health danger is hitting the health care system of the United States is the shortage of multiple drugs including opioid drugs as well (e.g.: fentanyl) necessitate the use of alternative supply channels (e.g.: importation of drugs outside the U.S., internet pharmacies) make the health sector more susceptible to illegal and counterfeit products.

Beside the network science the Big Data type of analysis is a novel and hopefully in the future a routinely used methodology to identify and monitor illegal vendors and their connections in the field of illegal internet sale of pharmaceuticals. The more detailed description of the method or even the representation with a figure would help the understanding of it.

The characterisation (e.g.: ordering is possible, LegitScript or VIPPS NABP legitimacy, products being sold, prescription requirements) of the identified online pharmacies would have highlighted the illegal internet sale of fentanyl products more accurately.

Moreover the inclusion of comparison of the results with the literature data regarding other social media platforms or the simple google search methodology would give a more complete picture of this public health danger. 
Is the work clearly and accurately presented and does it cite the current literature? Yes

Is the study design appropriate and is the work technically sound?

Yes

Are sufficient details of methods and analysis provided to allow replication by others? No

If applicable, is the statistical analysis and its interpretation appropriate? Yes

Are all the source data underlying the results available to ensure full reproducibility? Yes

Are the conclusions drawn adequately supported by the results?

Yes

Competing Interests: No competing interests were disclosed.

I confirm that I have read this submission and believe that I have an appropriate level of expertise to confirm that it is of an acceptable scientific standard.

Reviewer Report 30 November 2017

https://doi.org/10.5256/f1000research.14002.r27555

(C) 2017 Shepherd M. This is an open access peer review report distributed under the terms of the Creative Commons Attribution License, which permits unrestricted use, distribution, and reproduction in any medium, provided the original work is properly cited.

\section{Marvin D. Shepherd}

Division of Health Outcomes and Pharmacy Practice, College of Pharmacy, The University of Texas at Austin, Austin, TX, USA

My main concern is the discontinuity between the introduction and the research conducted. The introduction focused on problems of pharmaceutical purchased which were laced with counterfeit fentanyl. This is true and is a major health hazard as pointed out by the authors. Fentanyls are a deadly pharmaceuticals and again this was emphasized by the authors. However, the paper's research focus is on tweets from people who are interested in purchasing fentanyl illegally. It wasn't on the counterfeit fentanyl laced products. I suggest that the intro be rewritten and I mention the legitimate uses of fentanyl. Please mention that one of fentanyl's major uses is as a pain reliever in hospital surgical suites and must be carefully controlled by medical practitioners. To reiterate, the introduction doesn't match well the research content.

The methodology approach was was appropriate and innovative. I know they provided a link for a 
full description of the search terms, but in reading it I suggest that they provided at least a partial list of the names used i.e. different spellings of fentanyl, different names for the same product (foreign names). Searching tweets was original, but it didn't produce huge number of tweets. This is worth reporting in that few people tweet to purchase fentanyl. The first thing I thought of was why tweet when you can do a google search and get right to the web sites? This needs to be put in the discussion as to why the results were so low. I am sure there are other methods to find the product.

Is the work clearly and accurately presented and does it cite the current literature? Yes

Is the study design appropriate and is the work technically sound?

Yes

Are sufficient details of methods and analysis provided to allow replication by others? No

If applicable, is the statistical analysis and its interpretation appropriate?

Yes

Are all the source data underlying the results available to ensure full reproducibility? Yes

Are the conclusions drawn adequately supported by the results?

Yes

Competing Interests: No competing interests were disclosed.

I confirm that I have read this submission and believe that I have an appropriate level of expertise to confirm that it is of an acceptable scientific standard. 
The benefits of publishing with F1000Research:

- Your article is published within days, with no editorial bias

- You can publish traditional articles, null/negative results, case reports, data notes and more

- The peer review process is transparent and collaborative

- Your article is indexed in PubMed after passing peer review

- Dedicated customer support at every stage

For pre-submission enquiries, contact research@f1000.com 